20th ; cicatrisation complete; passes motions in small quantities frequently and with ease; a small quantity of pus still comes away from the anus, as well as mucus, which he thinks is diminishing.

\section{SYPHILITIC SORE ON THE FEMALE NIPPLE.}

(Under the care of Mr. Andrew Clark.)

M. A. S—, aged thirty-two, came as an out-patient to Mr. Clark in November last, with a sore, which had all the appearance of a chancre, on the left nipple. There was also an enlarged and tender gland in the axilla. The patient had always been a healthy woman, and her husband also was quite healthy; she remembered, however, putting a neighbour's child with a sore on its mouth to her breast about four months before, and, according to her own account, the sore formed two or three months afterwards. She was ordered to take perchloride of mercury, and to have black-wash applied to the chancre. In three weeks she was quite well, and ceased attending the hospital of her own accord.

On Dec. 31st she again attended, when she had a wellmarked syphilitic rash and the signs of secondary syphilis. She was again put under syphilitic treatment, and rapidly improved.

\section{ROYAL FREE HOSPITAL.}

\section{LIGATURE OF THE EXTERNAL ILIAC ARTERY FOR ANEURISM OF THE FEMORAL.}

(Under the care of Mr. JoHN D. HILL.)

Mr. HrLL, on the 23rd ult., tied the external iliac artery for aneurism of the femoral, situated just below Poupart's ligament. The operation is so far successful. A few minutes after the operation, the temperature of the limb fell to $89^{\circ} \mathrm{F}$. in the popliteal space, that of the axilla being $97^{\circ} \mathrm{F}$. In eight hours the temperature had regained its normal standard. The patient is progressing favourably. A full report of this case will appear at some future time.

\section{SUBCUTANEOUS OSTEOTOMY OF RADIUS FOR MALPOSI- TION AFTER FRACTURE; CURE.}

Mr. Hill also exhibited a patient in whom he had performed subcutaneous osteotomy of the shaft of the radius three months previously. It appears that some years ago the bone had been fractured, but the fragments had united in a false position, the upper part of the bone being supine, but the lower part prone. The patient was, moreover, the subject of rickets. The operation was rendered more than ordinarily difficult through the eburnation of the bone. Union of the fragments has now taken place in such a manner that supination and pronation of the forearm can readily be performed.

\section{SUNDERLAND INFIRMARY.}

We are indebted to Mr. Hopgood for the notes of the following cases:-

\section{FRACTURE OF THE PATELLA TREATED BY MALGATGNE'S HOOKS.}

(Under the care of Mr. Mongan.)

Michael F-, aged twenty-six, was admitted into the infirmary on Feb. 7th, 1872, suffering from fracture of the patella. There was great swelling about the knee shortly after adnission. A back splint was applied, and the fragments of the patella hrought as closely together as possible by means of a bandage applied in the form of a figure of $\mathbf{8}$, and an evaporating lotion kept constantly to it.

Feb. 21st.-Swelling nearly all gone. Malgaigne's hooks applied, and the fragments brought nearly together.

23rd.-Fragments brought together.

March 2od.-Has had no pain until to-day. The hooks to be left in, although there is a little inflammation round each point. Back splint to remain on.

3rd.-Small points of suppuration at the entrance of the hooks. To have poultice applied.

9th. - Hooks removed. Fragments close together, but there is not bony union. To be kept on back splint.
12th.-Back splint removed and starch bandage applied. Allowed to get up.

18th. - Starch bandage removed a short time every day, and knee slightly bent. Can get about very well.

30th.-Discharged cured. Allowed to walk out without starch bandage.

This case was much more satisfactory than it at first promised, the swelling being so great that the fragments could not be brought together; and the only reason why the union should not have been bony seems due to the timo between the accident and the application of the hooks.

\section{CONCUSSION OF THE BRAIN, FOLLOWED BY GIDDINESS} AND EPILEPTIC FITS.

(Under the care of Mr. MongaN.)

W. C- was admitted into the infirmary suffering from concussion of the brain, due to the falling of a large piece of wood from one of the coal-drops, which struck him on the back of the head and rendered him insensible, in which state be remained for twelve hours. There was no wound to the scalp, and no sign of depression.

Nov. 18th.-Complains of headache, and is unable to stand on account of feeling giddy. To take an ounce of white mixture every morning.

25th.- Sass that when he is in bed all things seem to go round with him.

28th.-Feels giddy; but he can now put on his own boots.

De. 3rd.-Is still dizzy, and cannot bear a hard hat on his head.

7th.-Can now walk about very well; but if he attempts to look up or put his tongue far out he becomes giddy. He left the hospital this day.

Feb. 4th.- Has continued to feel giddy at times; and once or twice he has had a fit-certainly once, for when holding a child he suddenly became unconscious, and dropped it, knowing nothing about what had happened. He became a dispensary patient on this date; and, upon questioning him, he said that on several occasions he found a fit coming on, and was obliged to fall down.

March 4th.- $\mathrm{He}$ has been taking bromide of potassium and belladonna; and under this treatment the fits have become less frequent, and he is now able to go to work.

This case seems to have been one of concussion, combined with some slight injury to the brain, and is interesting as an example of some of the injuries in railway accidents which give rise to symptoms which are of ten supposed to be feigned. In this case the man had no motive for shamming, and was only too anxious to go to work.

\section{BRADFORD INFIRMARY.}

\section{CASE OF PRIMARY CANCER OF THE OMENTUM, MESEN-} TERY, AND DIAPHRAGM.

(Under the care of Dr. Nrcou.)

THe following notes have been furnished by Mr. Roberts, housemhysician.

R. M-, aged sixty-three, labourer, admitted March $19 \mathrm{th}$ with great pain in the abdomen and ascites; very weak; looks cachectic.

No history was obtained as to cancer in the family. The illness ran the ordinary course of malignant cachexia, though the only physical sign was the feeling of a loose lump beneath the abdominal wall at the cæcum. He died on April 5th ; total duration of illness being three months.

Post mortem examination. - Except some appearance of cicarrices at the left pulmonary apex, and extensive adhesions of both lungs, nothing abnormal was found in the chest. The liver and spleen had their capsules rather white and thickened; kidneys about normal; pancreas, stomach, and bowels normal; but matting together the liver, diaphragm, stomach, spleen, pancreas, and large and small intestine, was a mass of hard cartilaginous-like matter, afterwards shown by the microscope to be cancer, infiltrated in to the mesentery, omentum, and diaphragm, and giving them a very knotted appearance. A rope of this matter, adberent to the opening of the inguinal canal, explained the above physical sign. One lump, with similar structure, was found in the peritoneal wall. The cancer appeared to be strictly primary to the omentum, mesentery, and diaphragm. 